\title{
Quiste hepático solitario congénito: diagnóstico prenatal Reporte de un caso
}

\author{
Eudes Gartner*; Jorge García**; Rodrigo Cifuentes***; Pedro Rovetto****
}

\begin{abstract}
RESUMEN: Se presenta un caso de quiste hepático solitario congénito gigante, con ecografía antenatal y cuyo rápido crecimiento se asocia al óbito fetal. Se revisa la literatura al respecto. es la primera publicación que se hace en la literatura científica hispana. Se consideran los diagnósticos diferenciales, la importancia del diagnóstico antenatal precoz, y el probable tratamiento.
\end{abstract}

\section{Resumen de historia clínica}

H.C. 1182549, hijo de Y.G

Madre de 27 años de edad, G2 A1 PO, con aborto espontáneo dos años antes. Embarazo actual controlado por M.D., con antecedente de Grupo materno B(-), sin anti-D al final del embarazo anterior. Ecografía obstétrica del 20/II/ 93, muestra feto normal de 25 semanas, sin anomalías. Es remitida la madre al H.U.V. el 19/III/93, por incremento exagerado de la altura uterina, con ecografía obstétrica en la cual reportan quiste abdominal de 9.4 x 7.6 x $6.9 \mathrm{cms}$, con feto único vivo en podálica para 29 ss de gestación, con polihidramnios (figura 1).

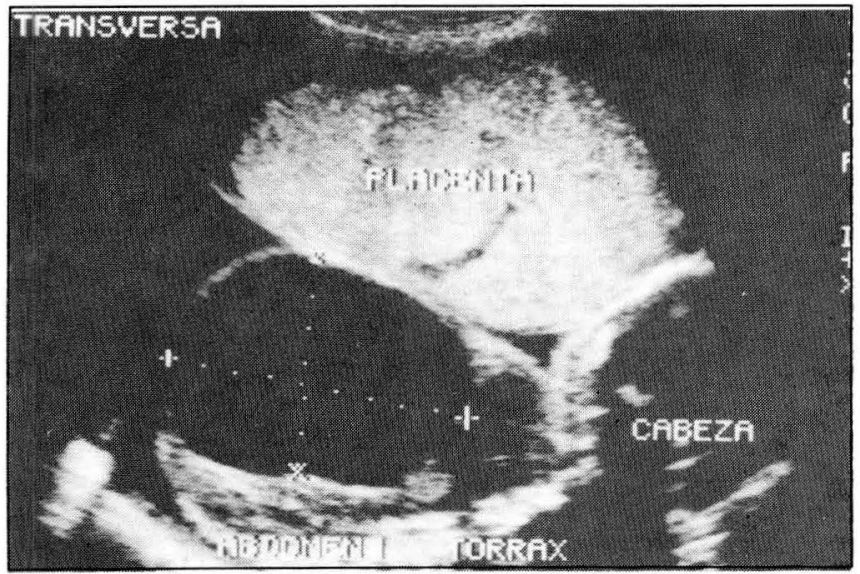

Figura 1

Quiste hepático

* Residente Neonatología.

** Residente Medicina Perinatal.

*** Profesor Titular - Jefe Medicina Perinatal. Universidad del Valle.

**** Profesor Titular - Departamento de Patología - Universidad del Valle.
$\mathrm{Al}$ ingreso de la paciente, con la historia referida, se encontró: T.A: 130/90, A.U. 29 cms, edema grado II en Ms.Is., F.C.F. 132; resto normal. Se ingresó con Dx de: Embarazo de 29 ss, con anomalía fetal; F.U.V. en podálica; polihidramnios y toxemia leve; posible isoinmunización $\mathrm{Rh}$.

Se practicó ecografía en urgencia, en la cual se corroboran hallazgos, y se solicita ecografía nivel III, la cual se programa para el 23/III/93.

La paciente hospitalizada evoluciona establemente, con vigilancia fetal que registra buenos movimientos y fetocardia, hasta el $23 / \mathrm{III} / 93$, cuando dejan de percibirse y se diagnostica óbito fetal. Se induce parto, el cual se obtiene 24 horas después con los siguientes hallazgos macroscópicos:

Feto sexo femenino, prematuro, con piel ligeramente esfacelada, sin ictericia.

Peso: 1600 grs. Talla $42 \mathrm{~cm}$. P.C. $26 \mathrm{~cm}$. P. abdominal $28,5 \mathrm{~cm}$. Examen externo: abdomen de aspecto en batracio, distendido. Resto normal para 29-30 semanas de edad gestacional.

Examen interno: derrame pleural bilateral, $50 \mathrm{cc}$ líquido serohemático a c/lado. Líquido ascítico sero-hemático, 150-180 cc.

Lesión quística hepática en el área del lóbulo derecho, el cual está atrófico, de $8 \mathrm{~cm}$ de diámetro, conteniendo líquido amarillo cetrino abundante, de paredes interiores arborescentes bien delimitadas, sin conexión aparente con conductos biliares; ausencia de vesícula biliar y vías biliares derechas (figura 2).

Vías biliares izquierdas dilatadas. Meconio teñido en intestino delgado y grueso. Demás órganos macroscópicamente normales.

\section{Descripción microscópica}

Cerebro: Congestión vascular

Pulmones: Colapsados, trombos de fibrina intravascular 


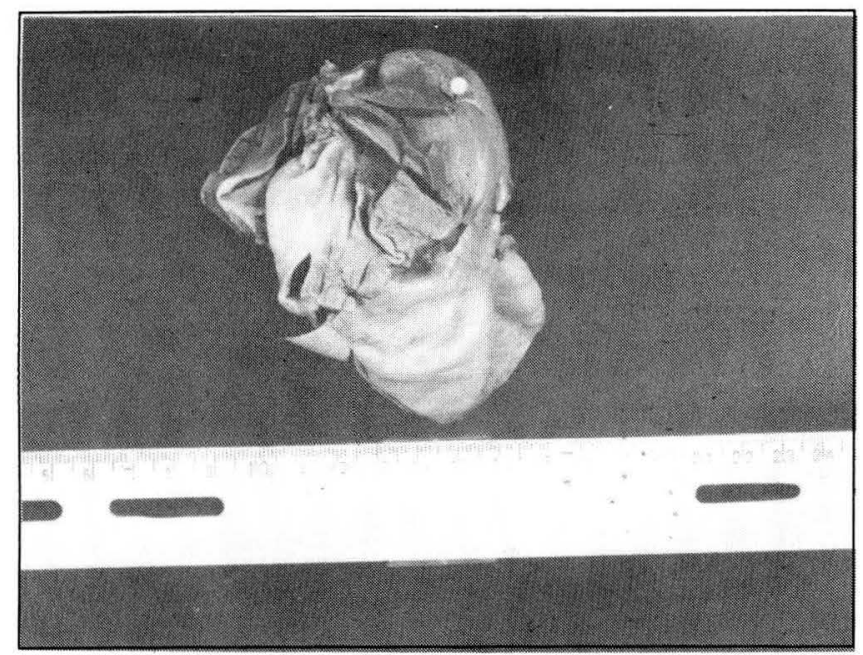

Figura 2

Aspecto macroscópico del hígado. A nivel del lóbulo derecho se observa formación quística de paredes blanquecinas con prominentes septos que le dan aspecto festoneado. (Cortesía Drs. H. Shultz., Cuello)

Riñones: Glomérulos alineados en corteza, congestión cortico-medular.

Hígado: Focos de heamatopoiesis extramedular. Colindando con parénquima normal se aprecia una pared quística formada por tejido hepático comprimido y tejido fibrovascular (figura 3)

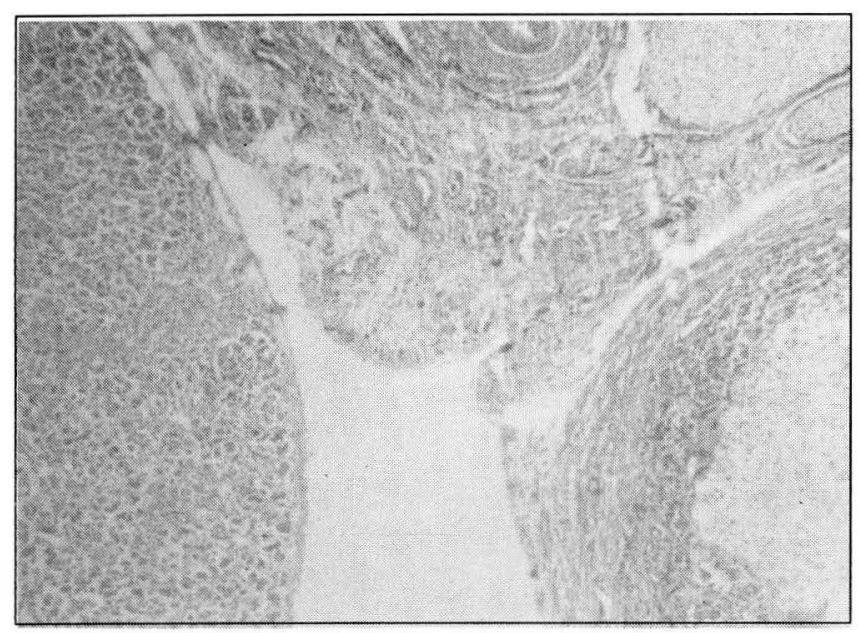

Figura 3

Corte histológico que representa parénquima hepático y pared quística conformada esta última por tejido hepático comprimido y tejido fibrovascular. (Cortesía Drs. H. Shultz., H. Cuello).

\section{Discusión}

El quiste hepático solitario congénito o no-parasitario se describió inicialmente en 1856 por Michel (13), siendo una entidad poco común. En revisión realizada por Byrne (7) en 1982, se encontraron cerca de 400 casos reportados hasta esa fecha; sin embargo, sólo 12 eran menores de 2 años, con 5 casos neonatales de los cuales 3 fallecieron por causa obstétrica relacionada con el tamaño del quiste.

Durante la última década han aumentado los reportes de lesiones quísticas intrahepáticas, apareciendo informes en la edad prenatal gracias al advenimiento de la ecografía de tiempo real y de alta resolución; en 1986 Chung (9) hace un primer reporte sobre diagnóstico ecográfico prenatal de esta patología.

El quiste hepático solitario congénito o no-parasitario, ha sido una entidad encontrada generalmente en la infancia o en la vida adulta, ya que la mayoría de las veces es pequeño y no produce sintomatología. En casos raros se ha asociado a muerte fetal por crecimiento rápido, como en nuestro paciente. Esto es igualmente válido para las diferentes entidades que producen lesiones quísticas en el hígado, y con las cuales se debe hacer diagnóstico diferencial.

El contenido del quiste usualmente es un líquido seroso claro y la pared que lo delimita es una capa celular originado del epitelio del tracto biliar. Su presentación es más frecuente en mujeres, puede ser intrahepático total o parcialmente y aún pedunculado, y es más frecuente en el lóbulo derecho que en el izquierdo. No se ha reportado asociación con malignidad, ni se acompaña de lesiones pancreáticas o renales, a diferencia de otras entidades con las cuales se realiza diagnóstico diferencial.

Recientemente se ha postulado que este tipo de lesiones serían un extremo de lo que se llamaría "continuum fibroquístico hepatobiliar", el cual incluiría quiste de colédoco, enfermedad de Caroli, síndromes poliquísticos y quiste único solitario (13).

El diagnóstico prenatal precoz es de suma importancia para identificar el quiste y tratarlo in útero con el objetivo de prevenir complicaciones fetales como daño hepático, oclusión de vías biliares, compresión del tubo digestivo y de le vena cava inferior, lo cual llevaría a alteraciones en funciones como la deglución de líquido amniótico y el gasto cardíaco, provocando a su vez polihidramnios y ascitis fetal, complicaciones éstas que empeoran aún más el pronóstico.

En relación con el manejo, Harrison en 1985 (11), empezó a realizar cirugía fetal intra-útero colocando catéteres "doble cola de cerdo" como tratamiento paliativo en quistes de otras cavidades serosas (pulmón y riñón en especial), con lo cual mejoró el pronóstico y la sobrevida mientras se realiza un tratamiento definitivo e el período neonatal temprano. Creemos que este tipo de manejo estaría indicado en los quistes hepáticos, como el descrito, descartando previamente con aspiración guiada por ultrasonido, comunicación con vías biliares, pues la presencia de bilis podría provocar peritonitis química en el feto. En el caso presente habría sido el manejo probable para mejorar pronóstico pues la simple aspiración no es suficiente, por la aparición de recidivas, como se ha demostrado en casos practicados en niños y adultos (3).

Finalmente, es de suma importancia realizar un diagnóstico diferencial adecuado (ver tabla 1), pues la mayoría de las entidades que producen lesiones quísticas en hígado, susceptibles de ser detectadas antenatalmente, requieren consejería genética para sus siguientes embarazos, ya que esta es una lesión que cursa sin alteraciones cromosómicas. 
Tabla 1

QUISTE HEPATICO SOLITARIO CONGENITO

\section{DIAGNOSTICO DIFERENCIAL}

- Enfermedad multiquística infantil

- Fibrosis hepática congénita

- Enfermedad poliquística infantil

- Otros síndromes asociados:

Meckel-Gruber

Ivemark

Zellwegwer

Distrofia torácica de Jeune

Elejalde

- Fibrosis nefronoptisis hepática congénita

(Nefronoptisis familiar de Fanconi)

- Displasia hepática ductal con: Esclerosis tuberosa

Atresia vaginal

Quistes renales medulares

- Enfermedad de Caroli simple

- Quiste de colédoco

- Dilatación biliar sistémica, o quiste de colédoco tipo V

\section{OTRAS ENTIDADES INTRAABDOMINALES}

- Quiste de ovario

- Duplicación quística

- Linfangioma quístico.
Finalmente, es de suma importancia realizar un diagnóstico diferencial adecuado (ver tabla 1), pues la mayoría de las entidades que producen lesiones quísticas en hígado, sušceptibles de ser detectadas antenatalmente, requieren consejería genética para sus siguientes embarazos, ya que esta es una lesión que cursa sin alteraciones cromosómicas.

\section{Agradecimientos}

Especiales agradecimientos al doctor Pedro Rovetto, docente de patología de la U. del Valle, quien revisó la autopsia y orientó la evaluación patológica del caso; doctor Harold Cuello, residente de patología de la U. del Valle, quien realizó el estudio de fotografía microscópica. Finalmente, reconocimiento muy particular a la participación de la doctora Heidi Schultz, quien llamó la atención sobre el caso patológico y su particularidad, y colaboró estrechamente en su evaluación.

\section{BIBLIOGRAFIA}

1. Walker WA., Durie PP. et al. Pediatric gastrointestinal diseases. Ed. B.C. Decker. 1991; 2: 1124-1129.

2. Colón AR. Textbook of pediatric patology, 2 Ed., 1990. Ed. Year Book Medical Publisher Inc. 192-194.

3. Wellwood JM. et al. Large intrahepatic cysts and pseudocysts. The american journal of surgery. Vol. 135, Jan. 1978; 57-64.

4. Sanjay y Saini et al. Percutaneous aspiration of hepatic cysts does not provide definitive therapy. Am. Jour. Roent. 1983; 141: 559-560.

5. Roemer CE. et al. Hepatic cysts: diagnosis and therapy by sonographic needle aspiration. A.J.R. 1981; 136: 1065-1070.

6. Terada Tadashi, Nakanuma Yasumi. Solitary cystic dilation of the intrahepatic bile duct. Am. Jour. Gast. Vol. 82, 12: 1987; 1301-1305

7. Byrne WJ., Fonkalsaud EW. Congenital solitary non parasitic cyst of the liver. A rare cause of rapidly enlarging abdominal mass in infancy. J. of Ped. Surg. 1982; 17: 316-317.
8. Grannum P.A. and Copel JA. Invasive fetal procedures. Fetal ultrasound. The radiologist Clinics of North America. 1990; 28: 217-226.

9. Chung WM. Antenatal detection of hepatic cysts. J. Clinic. Ultrasound 1986; 14: 217.

10. Callen RN. Ecografía en obstetricia y ginecología. Edit. Panamericana $2^{\mathrm{a}}$ edición $1991 ; 230-311$.

11. Harrison R., Colbus MS., Filly RA. The unborn patient. Prenatal diagnosis and treatment. 2nd edicion 1991-WB Sonders Company.

12. Geist DC. Solitary nonparasitic cyst of the liver. Arch. Surg. 1955; 71: 867-880.

13. Forbes A., Murray L. Cystic disease of the liver and biliary tract. Gut. 1991; Suppl: s116-122. 